\title{
GEODESY INSTRUMENT PACKAGE ON THE MOON FOR IMPROVING OUR KNOWLEDGE OF THE MOON AND THE REALIZATION OF REFERENCE FRAMES
}

\author{
V. DEHANT ${ }^{1}$, J. OBERST ${ }^{2}$, R. NADALINI ${ }^{3}$ \\ 1 Royal Observatory of Belgium \\ 3 avenue Circulaire, B1180 Brussels, Belgium \\ e-mail: v.dehant@oma.be \\ 2 Technical University Berlin and DLR-Berlin, Germany \\ e-mail: oberst@igg.tu-berlin.de \\ 3 Active Space Technologies GmbH, Berlin, Germany \\ e-mail: riccardo.nadalini@activespacetech.com
}

\begin{abstract}
The use of passive Laser reflectors on the lunar surface appeared to be the most attractive in the Apollo era, but the next generation of LLR experiment should therefore aim at a substantial improvement thanks to a one way ranging concept. A particular strength of this proposed experiment is given when several stations are ranging to the Moon simultaneously and/or when several geodetic stations on the Moon are used simultaneously, as this is expected to improve the modeling geometry and data quality. The proposed experiment may well initiate the installation of new observing stations on Earth perhaps within the infrastructure of existing astronomical observatories. In the case of the beacon mode, only passive optical receivers are needed on the ground. We explore the requirements for the instrumentation of such a ground station. We propose to deploy and operate a microwave receiver/transmitter with precisely known mechanical local ties to the Laser beacon/receiver, which will permit observations of the tangential position of the moon with respect to the celestial frame. We propose to include a GNSS microwave transmitter into the proposed equipment realizing a "GPS/Galileo satellite on the Moon" that is tracked together with GNSS satellites by receivers on the ground and possibly on the future generation of GNSS satellites. The ultimate objectives of our proposal are twofold, the improvement of the reference frames for the Earth and a better understanding of the Moon's interior.
\end{abstract}

\section{OBJECTIVES}

Owing to technological advances over the years, one can expect that the future geodetic measurement resolution will be improved by more than an order of magnitude. This step is nevertheless presently still limited by the instrumental precision level and by the geometry involved in the present measurement reference frames. We propose the deployment of a set of geodetic instruments on the Moon in order to improve the reference frame realizations for the Earth. In addition, the proposed set-up shall improve the ranging and Doppler measurements to the Moon, and thus the knowledge about the interior of the Moon. Applications to the moons of Mars and to lander on the Martian surface shall provide wonderful opportunities to dedicated science there and also to reference frame improvements.

\section{STATE OF THE ART AND NEXT DESIGN}

Presently there are only passive Laser reflectors on the lunar surface. These reflectors are targeted by the Earth Lunar Laser Ranging (LLR) ground stations and the round-trips of photons are used for measuring the Earth-Moon distance in the line of sight. There are presently five retroreflectors on the Moon surface.

A few decade ago, it was conceivable to make range measurements to the Moon by several alternative techniques. However, the use of passive Laser reflectors on the Lunar surface appeared to be the most attractive in the Apollo era for simple reasons: while the retroreflector arrays which had to be taken to the Moon were reasonably compact and very robust against failure, all the technologically complex instrumentation remained on Earth. In fact, owing to technological advances over the years, the measurement resolution could be improved by more than one order of magnitude, which is the basis of many 
scientific results that were achieved.

Unfortunately, the Laser link budget for ranging is very marginal, and even today, only very few terrestrial stations have the sophisticated technical equipment required to participate in Lunar ranging campaigns. As a consequence, a limited number of measurements have been collected in the past, and the distribution of measurements within the lunar cycle is strongly biased. A next generation LLR experiment should therefore aim at a substantial improvement of the link budget, so that the technique can cope e.g. with both a close proximity of the solar light background (new moon) and maximum brightness around the lunar target (full moon).

In parallel to this, it is highly desirable to complement the lunar laser ranging measurements with Very Long Baseline Interferometry (VLBI) measurements to the Moon as well as with the use of compact and precise radio transponders at the location of the landing sites on the Moon surface.

At the same time it is highly desirable to combine all the currently available high precision space geodetic techniques i.e. VLBI and Global Navigation Satellite System (GNSS) on Earth to match the currently available equipment at geodetic observatories. On Earth, the most commonly used precise positioning technique is indeed performed by GNSS observations. The GNSS satellite orbits are monitored from the ground and the inertial frame is provided by the observation of radiosources using VLBI. Similarly for the DORIS (Détermination d'Orbite et Radiopositionnement Intégrés par Satellite, or, in English, Doppler Orbitography and Radiopositioning Integrated by Satellite) observation, the Doppler and ranging measurements involved are used for obtaining positions and velocities of the beacon and of the spacecraft. In this context, it is very difficult to obtain precise inertial orbits for the GNSS constellation or for the DORIS system. Attempts are presently performed for a better precision of the orbits and of the Earth Orientation Parameters through combinations of the techniques. However it is very difficult to decontaminate the ground station tracking of the orbits from their own motion in space, inducing mapping of unmodeled effects in the orbits, which are changing with time.

As the Moon dynamics can be better followed than spacecraft undergoing wheel desaturation or maneuvers, we propose a new instrumental suite of technique combination on the Moon to obtain better ties between all reference frames in inertial space. This will provide in addition as very important objective the possibility to better understand the interior of the Moon. The suite involves a GNSS emitter, a microwave transponder, and an active laser system (see Figure 1).

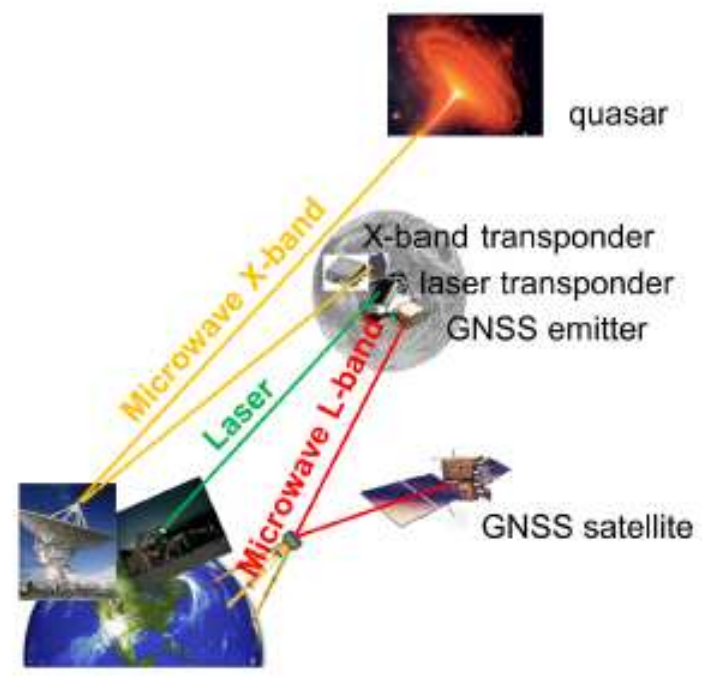

Figure 1: New proposed suite of instruments for a lander on the Moon.

This suite incorporates thus:

- An advanced Laser beacon / Laser receiver experiment to improve the Laser link budget substantially: while in the reflector case, the signal strength decreases with lunar distance $r$ to the fourth power $\left(1 / r^{4}\right)$, for transponders, signal strength decreases with only $1 / r^{2}$;

- A microwave receiver/transmitter (a coherent transponder); the down-link beacon (from the Moon to the Earth) will resemble quasar emission and can, thus, be observed in sequence with quasars in a differential mode; 
- A GNSS microwave transmitter (P-code); we practically realize a "GPS/Galileo satellite on the Moon" that is tracked together with GNSS satellites by receivers on the ground; the GPS/Galileo type of transmitter would make the Moon in its drag-free orbit a "natural GPS satellite" within the global navigation satellite system;

- A space qualified Cesium clock which will be the frequency reference for the whole suite, including the laser beacon generation and the GNSS or microwave;

- and additionally, one needs precisely known mechanical local ties between all the instrument and in particular to the Laser beacon.

\section{AIMS}

With the co-location of several geodetic techniques on the Moon, it is of course easier to understand the links between the techniques and to achieve better combinations of instruments. The results expected concerning the Moon position will be better. The combination will not only be distance observation and observation of velocity in the line-of-sight, but as well observations of the tangential position of the Moon with respect to the celestial frame. The system will not only allow better links between the techniques but as well allow to improve the modeling geometry and data quality. The proposed instrumentation realizes a "GPS/Galileo satellite on the Moon that is tracked together with GNSS satellites by receivers on the ground and possibly on the future generation of GNSS satellites.

The objectives will be summarized in the following:

- improvement of the reference frames for the Earth,

- better understanding of the Moons interior.

With this suite of instruments we are co-locating three different space geodetic techniques on the Moon. Each of these techniques is self-contained and draws on a different set of infrastructure on the Earth. The maximum benefit is expected when all the observations are coordinated between the techniques. Furthermore it may turn out that due to limitations of the available sensor block voltage supply the observations need to be scheduled by remote control for the optimum turnaround. An operation concept of the instrument set will be developed, and potential and limits of the experiment will be fully explored.

A Laser transponder on the Moon will greatly increase our knowledge on the variation of Lunar range. More measurements at higher accuracy, from observatories in both, Northern and Southern Hemisphere, without observational bias will be available. The new range data are the basis for comprehensive models of the dynamics of the Earth/Moon system and will give stronger determinations of Lunar ephemeris, rotation, and tidal deformation. They will also contribute to much better realizations of the various reference systems, i.e. the terrestrial and selenocentric frame, but also the dynamic realization of the celestial reference system. New reflectors on the Moon will provide additional accurate surface positions for cartographic control, and they also will contribute significantly to research in fundamental and gravitational physics.

The microwave transmitter/transponder will permit us to carry out observations of the tangential motion of the Moon in the quasi-inertial space with an accuracy in the range of 100 - 300 microarcseconds (which translates to the sub-meter level). It will also permit us to measure relative velocity of the Moon with respect to the Earth at better than a few hundredths of $\mathrm{mm} / \mathrm{s}$. Together with the radial information from Laser ranging, the tangential component from VLBI will push the insight into the Moon's orbital behavior including its libration to as yet unknown frontiers and there with obtain information on the core of the Moon). With the inclusion of a GNSS microwave transmitter in the package, three of the major space geodetic techniques will be co-located on the Lunar surface supporting the efforts for a rigorous combination of these techniques. This will eventually allow us to define a unified reference frame rather than a measurement-biased frame as today.

A "GPS/Galileo satellite on the Moon" or the "Moon as a natural GPS/Galileo satellite" that is linked to VLBI will make it possible to directly reference the GNSS satellite constellations (and thus GNSS-determined station coordinates) to the ICRF (International Celestial Reference Frame). This will allow us to measure UT1, i.e., the rotation angle of the Earth, as well as components of nutation with high temporal resolution (and there with obtain information on the deep interior of the Earth) while today GNSS is capable to provide only mean rates of Earth orientation and nutation.

A "local tie" between GNSS and VLBI on the Moon would allow for a consistent interpolation of VLBI derived UT1 data with high rate GNSS derived values. A link to the Moon may also improve 
the sensitivity of GNSS to components of the geocenter. The identification and monitoring of possible geocenter variations by GNSS is currently limited by satellite radiation pressure modeling deficiencies, a restriction that does not apply to the Earth's natural neighbor.

The possibility for continuous tracking of Lunar targets, independent of weather conditions and observation scheduling of LLR stations or large VLBI telescopes, is one of the major advantages of a GNSS transmitter on the Moon as well as of a microwave transmitter/transponder. It will provide additional independent comparisons, support calibration of measurements, and allow interpolation of SLR and VLBI results. In addition, such an instrument would allow for a continuous precise monitoring of the space clock.

These additional laser ranging measurements to the Moon will also allow better accuracy on the determination of the geophysical parameters such as the core dimension determined from libration and tidal displacements (Rambaux and Williams, 2010).

\section{CONCLUSIONS}

A GPS/Galileo satellite on the Moon or the Moon as a natural GPS/Galileo satellite that is linked to VLBI will make it possible to directly refer the GNSS satellite constellations (and thus GNSS-determined station coordinates) to the ICRF (International Celestial Reference Frame). Three of the major space geodetic techniques will be co-located on the lunar surface supporting the efforts for a rigorous combination of these techniques. The propose suite of instruments allows to combine all the currently available high precision space geodetic techniques, like VLBI, microwave at X-band, and GNSS at the location of the landing sites on the Moon, to match the currently available equipment at geodetic observatories on the Earth. With this approach, a unified reference frame will be computed rather than a measurement-biased frame.

Together with the radial information from Laser ranging (the position and velocity of the Moon with respect to the Earth will be determined at a never-reached precision of the sub-cm level and a few hundredths of $\mathrm{mm} / \mathrm{s}$ ), the tangential component from VLBI will push the insight into the Moon's orbital behaviour including its libration to as yet unknown frontiers and there with obtain information on the core of the Moon. This conclusion is further strengthened in the context the recent reprocessing of lunar seismic data and the findings related to a liquid core, a partial melting boundary layer, and a solid inner core inside the Moon.

Constraining the detailed structure of the lunar core is necessary to improve our understanding of the present-day thermal structure of the interior and the history of a lunar dynamo, as well as the origin and thermal and compositional evolution of the Moon.

\section{PERSPECTIVES; APPLICATION TO MARS}

The described system design developed for the Moon, can be adapted to Phobos and Deimos, the two moons of Mars, or to landers on Mars. Particular efforts will have to be performed for respecting the link budgets. Geodetic experiments in the Mars system will allow us to constrain the interiors of Mars and its moons, study the origin of the Mars satellite system, and even testing relativity theory. There are presently two mission proposed respectively for the Exploration Programme and Science Programme of ESA: the Mars-GeO and GETEMME.

1. Mars-GeO (for Mars Geophysical Observatories) mission to Mars (with an orbiter and landers in order to study the interior of Mars); this proposal is now considered for Exploration Programme and not for the Science Programme anymore; Veronique Dehant PI.

2. GETEMME (Gravity, Einstein's Theory, and Exploration of the Martian Moons' Environment) proposal (aiming at precise relative position of Phobos and Deimos in order to solve the question of their interior structure and origin); this proposal has been sent in with laser and radioscience instruments; Juergen Oberst PI.

\section{REFERENCES}

Rambaux, N., Williams J.G., 2010, The Moon's physical librations and determination of their free modes, Celestial Mechanics and Dynamical Astronomy, DOI: 10.1007/s10569-010-9314-2. 